\title{
The classification of nonsingular multidimensional Dubrovin-Novikov brackets 1
}

\author{
O. I. Mokhov \\ Centre for Nonlinear Studies, L.D.Landau Institute for Theoretical Physics, \\ Russian Academy of Sciences, Kosygina 2, Moscow, 117940, Russia \\ Department of Geometry and Topology, Faculty of Mechanics and Mathematics, \\ M.V.Lomonosov Moscow State University, Moscow, 119992, Russia \\ e-mail:mokhov@mi.ras.ru; mokhov@landau.ac.ru; mokhov@bk.ru
}

To the memory of my wonderful mother

Maya Nikolayevna Mokhova (04.05.1926 - 12.09.2006)

\begin{abstract}
In this paper the well-known Dubrovin-Novikov problem posed as long ago as 1984 in connection with the Hamiltonian theory of systems of hydrodynamic type, namely, the classification problem for multidimensional Poisson brackets of hydrodynamic type, is solved. In contrast to the one-dimensional case, in the general case, a nondegenerate multidimensional Poisson bracket of hydrodynamic type cannot be reduced to a constant form by a local change of coordinates. Generally speaking, such a Poisson bracket is generated by a nontrivial special infinite-dimensional Lie algebra. In this paper we obtain a classification of all nonsingular nondegenerate multidimensional Poisson brackets of hydrodynamic type for any number $N$ of components and for any dimension $n$ by differential-geometric methods. A key role in the solution of this problem was played by the theory of compatible metrics that had been earlier constructed by the present author.
\end{abstract}

\footnotetext{
${ }^{1}$ The research was supported by the Max-Planck-Institut für Mathematik (Bonn, Germany), the Russian Science Support Foundation, the Russian Foundation for Basic Research (grants Nos. 0301-00782 and 05-01-00170), and the Program of Support for Leading Scientific Schools (grant No. NSh-4182.2006.1).
} 
Key words and phrases: multidimensional Dubrovin-Novikov bracket, multidimensional Poisson bracket of hydrodynamic type, local pseudo-Riemannian geometry, obstruction tensor, infinite-dimensional Lie algebra, compatible and almost compatible pseudo-Riemannian metrics, flat pencil of metrics, Hamiltonian system, system of hydrodynamic type, compatible Poisson brackets, Nijenhuis tensor, linear Poisson bracket, Frobenius and quasi-Frobenius algebra.

AMS 2000 Mathematics Subject Classification: 35F20, 35Q35, 37K05, 37K10, 37K25, 37K30, 53B20, 53B30.

\section{Introduction}

In this paper the well-known Dubrovin-Novikov problem is solved. This is the problem, posed as long ago as 1984 in [1], of the classification of multidimensional Poisson brackets of hydrodynamic type, i.e., field-theoretic Poisson brackets of the form

$$
\left\{u^{i}(x), u^{j}(y)\right\}=\sum_{\alpha=1}^{n}\left(g^{i j \alpha}(u(x)) \delta_{\alpha}(x-y)+b_{k}^{i j \alpha}(u(x)) u_{\alpha}^{k}(x) \delta(x-y)\right),
$$

where $u=\left(u^{1}, \ldots, u^{N}\right)$ are local coordinates on a certain smooth $N$-dimensional manifold $M$ or in a domain of $\mathbf{R}^{N} ; x=\left(x^{1}, \ldots, x^{n}\right), y=\left(y^{1}, \ldots, y^{n}\right)$ are independent variables; the coefficients $g^{i j \alpha}(u), b_{k}^{i j \alpha}(u)$ are smooth functions of the local coordinates $\left(u^{1}, \ldots, u^{N}\right), 1 \leq i, j, k \leq N, 1 \leq \alpha \leq n ; u(x)=\left(u^{1}(x), \ldots, u^{N}(x)\right)$ are smooth functions ( $N$-component fields) of $n$ independent variables $x^{1}, \ldots, x^{n}$ with values in the manifold $M ; u_{\alpha}^{k}(x)=\partial u^{k} / \partial x^{\alpha}$; and $\delta(x)$ is the Dirac delta-function, $\delta_{\alpha}(x-y)=$ $\partial \delta(x-y) / \partial x^{\alpha}$.

The condition of skew-symmetry and the Jacobi identity for a Poisson bracket of the form (1.1) impose very severe restrictions on the coefficients $g^{i j \alpha}(u)$ and $b_{k}^{i j \alpha}(u)$ (we shall talk about these restrictions below).

The general class of Poisson brackets of the form (1.1) was introduced by B.A.Dubrovin and S.P.Novikov in [2] (the one-dimensional case $n=1$ ) and [1] (the multidimensional case of arbitrary $n$ ) in connection with the Hamiltonian theory of systems of hydrodynamic type, and such Poisson brackets are also called by multidimensional Dubrovin-Novikov brackets. The very natural differential-geometric Hamiltonian approach to systems of hydrodynamic type, i.e., evolution systems of homogeneous quasilinear equations with partial derivatives of the first order, proposed in [1] and [2], is connected with the general class of Poisson brackets of the form (1.1). 
If $\operatorname{det}\left(g^{i j \alpha}(u)\right) \neq 0$ for all $\alpha$, then the bracket (1.1) is called nondegenerate. The one-dimensional nondegenerate Poisson brackets of hydrodynamic type were completely classified by B.A.Dubrovin and S.P.Novikov in 1983 in [2], where they proved that each such Poisson bracket can be reduced to a constant form by a local change of coordinates and can be characterized by a unique invariant, namely, by signature of the metric of the bracket.

Afterwards this Hamiltonian approach led to the creation of the remarkable and very fruitful theory of integrable one-dimensional systems of hydrodynamic type (see [3], 4]), and at present one can consider that the Hamiltonian and integrability properties of one-dimensional systems of hydrodynamic type are well studied (although in the one-dimensional case also many very important problems remain still unsolved).

However, the case of multidimensional systems of hydrodynamic type is considerably more complicated and, in fact, has not been studied as yet from the viewpoint of the general Hamiltonian and integrability properties (see [5]). In the multidimensional case, very serious problems arise already under studying the simplest natural invariant class of suitable local Hamiltonian structures (1.1).

In [1] B.A.Dubrovin and S.P.Novikov posed the classification problem for nondegenerate multidimensional Poisson brackets of the form (1.1) and they showed therewith that for the multidimensional brackets, in contrast to one-dimensional, the statement on reducing to a constant form by a local change of coordinates is definitely not true, since, in particular, the two-dimensional nondegenerate Poisson bracket of hydrodynamic type, generated by the Lie algebra of vector fields on the plane and related to the two-dimensional Euler hydrodynamics, cannot be reduced to a constant form by a local change of coordinates (there are nonzero tensor obstructions in this case). In the same paper [1] B.A.Dubrovin and S.P.Novikov undertook an unsuccessful attempt to obtain a classification of these brackets in the case $N=1$ and in the first nontrivial case $n=N=2$ (it was shown in [6] by the author of the present paper that these results in the paper [1] were erroneous).

A complete classification of all one-component $(N=1, n$ arbitrary) and all nondegenerate two-component $(N=2, n$ arbitrary) Poisson brackets of the form (1.1) was obtained by the present author in [6], where a complete classification was also obtained for $1 \leq N \leq 4$, for which it was necessary to develop powerful algebraic machinery, but all attempts to solve the problem for an arbitrary number $N$ of components by algebraic methods have so far been unsuccessful.

In the general case, a nondegenerate multidimensional Poisson bracket of hydrodynamic type cannot be reduced to a constant form by a local change of coordinates, in contrast to the one-dimensional case, but according to results of B.A.Dubrovin and 
S.P.Novikov [1], and also the present author [6], for any such Poisson bracket, there always exist special local coordinates such that the bracket is linear with respect to the fields (generally speaking, linear nonhomogeneous).

Multidimensional Poisson brackets of hydrodynamic type that are linear with respect to the fields are generated by special infinite-dimensional Lie algebras (Lie algebras of hydrodynamic type) and special 2-cocycles on these infinite-dimensional Lie algebras (for nonhomogeneous linear brackets). A classification of these Lie algebras for $n \geq 2$ was obtained for the cases $1 \leq N \leq 4$ by the author of the present paper in [6]. (One-dimensional Dubrovin-Novikov brackets that are linear with respect to the fields and algebraic structures related to them were studied in [7].)

The classification problem for multidimensional nondegenerate Poisson brackets of hydrodynamic type, for an arbitrary number of components, was absolutely inaccessible and inapproachable up to now. In this paper we obtain a classification of all nonsingular nondegenerate multidimensional Poisson brackets of the form (1.1) (in any case, for the situation of brackets of the form (1.1) in general position) for arbitrary number $N$ of components and for arbitrary $n$ by differential-geometric methods. A key role in the solution of this problem was played by the theory of compatible metrics constructed by the present author in [8].

\section{Multidimensional homogeneous brackets of the first order and Dubrovin-Novikov brackets}

The requirement of bilinearity and the fulfilment of the Leibniz identity for a bracket

of the form (1.1), i.e., an arbitrary multidimensional homogeneous bracket of the first order, is equivalent to the condition that on arbitrary functionals $I$ and $J$ on the space of fields $u(x)$ the bracket has the form

$$
\{I, J\}=\sum_{\alpha=1}^{n} \int \frac{\delta I}{\delta u^{i}(x)}\left(g^{i j \alpha}(u(x)) \frac{d}{d x^{\alpha}}+b_{k}^{i j \alpha}(u(x)) u_{\alpha}^{k}(x)\right) \frac{\delta J}{\delta u^{j}(x)} d^{n} x,
$$

where $d / d x^{\alpha}$ is the total derivative with respect to the independent variable $x^{\alpha}$,

$$
\begin{gathered}
\frac{d}{d x^{\alpha}}=\frac{\partial}{\partial x^{\alpha}}+u_{\alpha}^{i} \frac{\partial}{\partial u^{i}}+u_{\beta \alpha}^{i} \frac{\partial}{\partial u_{\beta}^{i}}+\cdots+u_{\beta_{1} \cdots \beta_{s} \alpha}^{i} \frac{\partial}{\partial u_{\beta_{1} \cdots \beta_{s}}^{i}}+\cdots, \\
u_{\beta_{1} \cdots \beta_{s}}^{i}=\frac{\partial^{s} u^{i}}{\partial x^{\beta_{1}} \cdots \partial x^{\beta_{s}}},
\end{gathered}
$$


where summation over repeating upper and lower indices is assumed, moreover, in this case the summation over $\beta_{1}, \ldots, \beta_{s}$ is taken over distinct (up to arbitrary permutations) sets of these indices (one can consider that these sets of indices are ordered: $\left.\beta_{1} \leq \cdots \leq \beta_{s}\right)$.

The class of brackets of the form (2.1) is invariant with respect to local changes of coordinates $\left(u^{1}, \ldots, u^{N}\right)$. Here for each $\alpha$ the coefficients $g^{i j \alpha}(u)$ and $b_{k}^{i j \alpha}(u)$ of the brackets are transformed as differential-geometric objects. For any bracket of the form (2.1) the coefficient $g^{i j \alpha}(u)$ for each $\alpha$ is a contravariant two-valent tensor on the manifold $M$. For any nondegenerate bracket of the form (2.1) and for each $\alpha$ we can introduce coefficients $\Gamma_{i j}^{k \alpha}(u)$ by the formulae $\Gamma_{i j}^{k \alpha}(u)=-g_{i s}^{\alpha}(u) b_{j}^{s k \alpha}(u)$, where $g_{i s}^{\alpha}(u)$ is a covariant two-valent tensor inverse to the tensor $g^{i j \alpha}(u), g^{i j \alpha}(u) g_{j k}^{\alpha}(u)=\delta_{k}^{i}$. Here for any nondegenerate bracket of the form (2.1) and for each $\alpha$ the coefficients $\Gamma_{s k}^{j \alpha}(u)$ are transformed as the coefficients of an affine connection on the manifold $M$. Thus a nondegenerate bracket of the form (2.1) is given by $n$ arbitrary nondegenerate contravariant two-valent tensors $g^{i j \alpha}(u), 1 \leq \alpha \leq n$, and $n$ arbitrary affine connections $\Gamma_{s k}^{j \alpha}(u), 1 \leq \alpha \leq n$, on the manifold $M$.

Note that among brackets of the form (2.1) there are, in particular, very simple, but very important brackets that do not depend on the fields $u(x)$ at all, namely, constant brackets (brackets of a constant form). All such brackets are given by the conditions $g^{i j \alpha}(u)=$ const, $b_{k}^{i j \alpha}(u)=0$. The problem, which we solve in this paper, is to find what the simplest canonical forms can be reduced Poisson brackets of the form (2.1) by local changes of coordinates to.

First of all, we shall show the general relations on the coefficients of Poisson brackets of the form (2.1) [9].

Theorem 2.1 [9]. A bracket (2.1) is a Poisson bracket, i.e., is skew-symmetric and satisfies the Jacobi identity, if and only if the following relations for the coefficients of the bracket are fulfilled:

$$
\begin{gathered}
g^{i j \alpha}=g^{j i \alpha}, \\
\frac{\partial g^{i j \alpha}}{\partial u^{k}}=b_{k}^{i j \alpha}+b_{k}^{j i \alpha} \\
\sum_{(\alpha, \beta)}\left(g^{s i \alpha} b_{s}^{j r \beta}-g^{s j \beta} b_{s}^{i r \alpha}\right)=0, \\
\sum_{(i, j, r)}\left(g^{s i \alpha} b_{s}^{j r \beta}-g^{s j \beta} b_{s}^{i r \alpha}\right)=0,
\end{gathered}
$$




$$
\begin{gathered}
\sum_{(\alpha, \beta)}\left[g^{s i \alpha}\left(\frac{\partial b_{s}^{j r \beta}}{\partial u^{q}}-\frac{\partial b_{q}^{j r \beta}}{\partial u^{s}}\right)+b_{s}^{i j \alpha} b_{q}^{s r \beta}-b_{s}^{i r \alpha} b_{q}^{s j \beta}\right]=0, \\
g^{s i \beta} \frac{\partial b_{q}^{j r \alpha}}{\partial u^{s}}-b_{s}^{i j \beta} b_{q}^{s r \alpha}-b_{s}^{i r \beta} b_{q}^{j s \alpha}=g^{s j \alpha} \frac{\partial b_{q}^{i r \beta}}{\partial u^{s}}-b_{s}^{j i \alpha} b_{q}^{s r \beta}-b_{q}^{i s \beta} b_{s}^{j r \alpha}, \\
\frac{\partial}{\partial u^{k}}\left[g^{s i \alpha}\left(\frac{\partial b_{s}^{j r \beta}}{\partial u^{q}}-\frac{\partial b_{q}^{j r \beta}}{\partial u^{s}}\right)+b_{s}^{i j \alpha} b_{q}^{s r \beta}-b_{s}^{i r \alpha} b_{q}^{s j \beta}\right]+ \\
+\sum_{(i, j, r)}\left[b_{q}^{s i \beta}\left(\frac{\partial b_{k}^{j r \alpha}}{\partial u^{s}}-\frac{\partial b_{s}^{j r \alpha}}{\partial u^{k}}\right)\right]+ \\
+\frac{\partial}{\partial u^{q}}\left[g^{s i \beta}\left(\frac{\partial b_{s}^{j r \alpha}}{\partial u^{k}}-\frac{\partial b_{k}^{j r \alpha}}{\partial u^{s}}\right)+b_{s}^{i j \beta} b_{k}^{s r \alpha}-b_{s}^{i r \beta} b_{k}^{s j \alpha}\right]+ \\
+\sum_{(i, j, r)}\left[b_{k}^{s i \alpha}\left(\frac{\partial b_{q}^{j r \beta}}{\partial u^{s}}-\frac{\partial b_{s}^{j r \beta}}{\partial u^{q}}\right)\right]=0 .
\end{gathered}
$$

Relations (2.3) and (2.4) are equivalent to the skew-symmetry of a bracket (2.1), and relations (2.5)-(2.9) are equivalent to the fulfilment of the Jacobi identity for a skewsymmetric bracket of the form (2.1).

We do not assume nondegeneracy of brackets and do not impose any additional conditions on the coefficients of brackets (2.1) in Theorem 2.1. Moreover, this theorem remains true for the important case (for example, in the theory of nonlinear chains), when the indices run an infinite set of values (for example, the sets of all integer or natural numbers) and any function under consideration (i.e., all the coefficients of brackets) depends on an arbitrary, but finite, number of the variables (in the onedimensional case, a similar observation was made by I.Dorfman in [10]). The signs $\sum_{(\alpha, \beta)}$ and $\sum_{(i, j, k)}$ mean summations over all cyclic permutations of the indicated indices, i.e., in the given case, the indices $(\alpha, \beta)$ and $(i, j, k)$ respectively. In the onedimensional case the general relations on the coefficients of the Poisson brackets of hydrodynamic type (without the assumption of nondegeneracy) were shown in [11], where there is an error in the formulae, corrected in [12] under construction of the nonlocal generalization of the one-dimensional Dubrovin-Novikov brackets.

In particular, the following important lemma immediately follows from the relations of Theorem 2.1. 
Lemma 2.1 For each multidimensional Poisson bracket of the form (2.1) and for each $\alpha$ the corresponding summand on the right-hand side of the formula (2.1) is a one-dimensional Poisson bracket of hydrodynamic type, i.e., each multidimensional Poisson bracket of the form (2.1) is always the sum of one-dimensional Poisson brackets with respect to each of the independent variables $x^{\alpha}$.

Besides, in the multidimensional case, for $n \geq 2$, the relations of Theorem 2.1 impose additional nontrivial restrictions on the coefficients of these one-dimensional Poisson brackets.

First of all, let us consider the relations (2.3)-(2.9) in the one-dimensional case (for $n=1$ ). It is obvious that in the one-dimensional case the relation (2.6) follows from the relation (2.5) and, moreover, in this case the relation (2.8) follows from the relations (2.7), (2.5) and (2.4). In the nondegenerate one-dimensional case $\left(\operatorname{det}\left(g^{i j \alpha}(u)\right) \neq 0, x=x^{\alpha}\right)$ we have: the relation (2.3) gives the condition that a nondegenerate tensor $g^{i j \alpha}$ is symmetric, i.e., $g^{i j \alpha}$ is a Riemannian or pseudo-Riemannian contravariant metric on the manifold $M$; the relation (2.4) means that the connection $\Gamma_{i j}^{k \alpha}=-g_{i s}^{\alpha} b_{j}^{s k \alpha}$ is compatible with the metric $g^{i j \alpha}$, i.e., the corresponding covariant derivative of the metric is equal to zero; the relation (2.5) is equivalent to the condition that the connection $\Gamma_{i j}^{k \alpha}$ is symmetric, i.e., $\Gamma_{i j}^{k \alpha}=\Gamma_{j i}^{k \alpha}$; the relation (2.7) means exactly that the connection is flat, i.e., the Riemannian curvature tensor vanishes; the relation (2.9) for $\alpha=\beta$ and for a nondegenerate metric $g^{i j \alpha}$ follows from the relations (2.5) and (2.7).

This proves the Dubrovin-Novikov theorem [2]: an arbitrary nondegenerate onedimensional Poisson bracket of hydrodynamic type is uniquely determined by an arbitrary flat metric $g^{i j}(u)$ and is reduced to a constant form in any flat coordinates of this metric, and $\Gamma_{i j}^{k}(u)=-g_{i s}(u) b_{j}^{s k}(u)$ is the Levi-Civita connection generated by the metric $g^{i j}(u)$.

Thus, by virtue of Lemma 2.1 and the Dubrovin-Novikov theorem for nondegenerate one-dimensional Poisson brackets of the form (1.1) [2], all the tensors $g^{i j \alpha}(u)$ are flat metrics (metrics of zero Riemannian curvature), and each the corresponding affine connection $\Gamma_{s k}^{j \alpha}(u)$ is compatible with the respective metric $g^{i j \alpha}(u)$ and has zero torsion and zero Riemannian curvature, i.e., the affine connection $\Gamma_{s k}^{j \alpha}(u)$ is the Levi-Civita connection and is uniquely determined by the flat metric $g^{i j \alpha}(u)$.

Hence, each nondegenerate Poisson bracket of the form (2.1) is uniquely determined by the flat metrics $g^{i j \alpha}(u)$, which are connected by additional severe restrictions in the multidimensional case, and our problem is reduced to a classification of the admissible sets of the flat metrics $g^{i j \alpha}(u), 1 \leq \alpha \leq n$. 


\section{Tensor obstructions and tensor relations}

Let us consider an arbitrary nondegenerate multidimensional Poisson bracket of the form (2.1) and introduce the tensors $T_{j k}^{i \alpha \beta}(u)=\Gamma_{j k}^{i \beta}(u)-\Gamma_{j k}^{i \alpha}(u)$ defined for each pair of distinct indices $\alpha$ and $\beta$. The tensors $T_{j k}^{i \alpha \beta}(u)$ are obstructions for reducing nondegenerate multidimensional Poisson brackets of the form (2.1) to constant brackets, i.e., an arbitrary nondegenerate Poisson bracket of the form (2.1) can be reduced to a constant bracket by a local change of coordinates if and only if all the obstruction tensors $T_{j k}^{i \alpha \beta}(u)$ are identically equal to zero.

Indeed, if even one of these tensors is not equal to zero identically, then the Poisson bracket cannot be reduced to a constant form by a local change of coordinates, since for any constant bracket the coefficients of all the connections $\Gamma_{j k}^{i \alpha}(u)$ are equal to zero identically, and, consequently, in these coordinates all the tensors $T_{j k}^{i \alpha \beta}(u)$ should be equal to zero identically. The converse statement is also obvious. Indeed, if all the tensors $T_{j k}^{i \alpha \beta}(u)$ are equal to zero identically, then all the connections $\Gamma_{j k}^{i \alpha}(u)$ are equal one to another and, consequently, all of them are equal to zero in any flat coordinates of the metric $g^{i j 1}(u)$, and all the metrics of the bracket are necessarily constant in these coordinates by virtue of compatibility of the metrics with the corresponding connections.

The following theorem gives a complete set of tensor relations defining the class of nondegenerate Poisson brackets of the form (2.1).

Theorem 3.1 [6]. Flat nondegenerate metrics $g^{i j \alpha}(u)$ define a multidimensional Poisson bracket of the form (2.1) if and only if the following relations are fulfilled:

$$
\begin{gathered}
T^{i j k \alpha \beta}(u)=T^{k j i \alpha \beta}(u), \\
\sum_{(i, j, k)} T^{i j k \alpha \beta}(u)=0, \\
T^{i j s \alpha \beta}(u) T_{s t}^{r \alpha \beta}(u)=T^{i r s \alpha \beta}(u) T_{s t}^{j \alpha \beta}(u), \\
\nabla_{r}^{\alpha} T^{i j k \alpha \beta}(u)=0,
\end{gathered}
$$

where $T_{j k}^{i \alpha \beta}(u)=\Gamma_{j k}^{i \beta}(u)-\Gamma_{j k}^{i \alpha}(u), T^{i j k \alpha \beta}(u)=g^{k s \beta}(u) g^{i r \alpha}(u) T_{r s}^{j \alpha \beta}(u)$, the sign $\sum_{(i, j, k)}$ means summation over all cyclic permutations of indices $(i, j, k), \nabla_{r}^{\alpha}$ is the covariant derivative given by the connection $\Gamma_{j k}^{i \alpha}(u)$, and $\Gamma_{j k}^{i \alpha}(u)$ is the Levi-Civita connection generated by the metric $g^{i j \alpha}(u)$. 
The relations (3.1) and (3.3) were found by B.A.Dubrovin and S.P.Novikov in [1]. It was proved by the present author in [6] that this set of tensor relations is not complete, and the complete system of tensor relations (3.1)-(3.4) for nondegenerate Poisson brackets of the form (2.1) was obtained [6]. The relations (3.2) and (3.4) are very essential and play an important role under classifying the multidimensional Poisson brackets of hydrodynamic type.

If all the metrics $g^{i j \alpha}$ are nondegenerate, then we obtain from Theorem 2.1 that for $\alpha \neq \beta$ the condition (2.5) gives the relation (3.1) for the obstruction tensors; the condition (2.6) is equivalent to the relation (3.2); the condition (2.7) is equivalent to the relation (3.3); the condition (2.8) is equivalent to the relation (3.4); the condition (2.9) for nondegenerate metrics is a direct consequence of the relations (2.3)-(2.8) (this is not true in the general case and so the condition (2.9) is essential in the case of degenerate metrics).

It is not complicated to obtain a complete classification of all one-component (degenerate and nondegenerate) Poisson brackets of the form (2.1). Let us prove that for $N=1$ and arbitrary $n$ all the obstruction tensors are identically equal to zero. Indeed, in the one-component case the relations (2.3), (2.5), (2.7) and (2.9) are automatically fulfilled, the relation (2.4) gives $\partial g^{\alpha} / \partial u=2 b^{\alpha}(u)$, from the relation (2.6) we obtain that $g^{\alpha}(u) b^{\beta}(u)=g^{\beta}(u) b^{\alpha}(u)$, and the relation (2.8) follows from (2.4) and (2.6). Thus in the one-component case for any $n$ and for any indices $\alpha$ and $\beta$ we have $\Gamma^{\alpha}(u)=-b^{\alpha}(u) / g^{\alpha}(u)=-b^{\beta}(u) / g^{\beta}(u)=-\left(1 /\left(2 g^{\alpha}(u)\right)\right) \partial g^{\alpha} / \partial u=$ $-\left(1 /\left(2 g^{\beta}(u)\right)\right) \partial g^{\beta} / \partial u=\Gamma^{\beta}(u)$, i.e., $g^{\alpha}(u)=c^{\alpha} g(u)$, where $g(u)$ is an arbitrary nonzero function, and $c^{\alpha}$ are arbitrary nonzero constants. Hence, all the obstruction tensors $T^{\alpha \beta}(u)=\Gamma^{\beta}(u)-\Gamma^{\alpha}(u)$ are identically equal to zero, and each multidimensional one-component Poisson bracket of the form (2.1) is reduced to a constant form by a local change of the unique coordinate $u=u^{1}$ (in the degenerate case, i.e., if

$g^{\alpha}(u)=0$, then also $b^{\alpha}(u)=0$ by virtue of the relation (2.4) $)$. Hence, we obtain a complete classification of all one-component (degenerate and nondegenerate) Poisson brackets of the form (2.1).

\section{Linear Poisson brackets of hydrodynamic type, infinite-dimensional Lie algebras, Frobenius and quasi-Frobenius algebras}

Each flat metric $g^{i j \alpha}(u)$ can be reduced to a constant form by a local change of coordinates. Let us reduce one of the metrics (for definiteness, we reduce the first 
one) of an arbitrary nondegenerate Poisson bracket of the form (2.1) to a constant form.

Theorem 4.1 If for a nondegenerate multidimensional Poisson bracket of the form (2.1) we have $g^{i j 1}=$ const, then either this Poisson bracket is constant in these local coordinates, or it has nonzero obstruction tensors, i.e., this Poisson bracket cannot be reduced to a constant form by local changes of coordinates at all.

Indeed, if in the given coordinates $\Gamma_{j k}^{i \alpha}=0$ for all $\alpha$, then $b_{k}^{i j \alpha}=0$ for all $\alpha$ and the Poisson bracket is constant by virtue of the relation (2.4). But if for a certain $\alpha$ not all of the coefficients of the connection $\Gamma_{j k}^{i \alpha}$ are equal to zero, then also the obstruction tensor $T_{j k}^{i 1 \alpha}$, which coincides in the given local coordinates with $\Gamma_{j k}^{i \alpha}$, is not equal to zero, and, consequently, this Poisson bracket cannot be reduced to a constant form by local changes of coordinates.

The following important theorem is a simple consequence of Theorem 3.1 .

Theorem 4.2 [1], 6]. If $g^{i j 1}=$ const for a nondegenerate multidimensional Poisson bracket of the form (2.1), then all the other metrics are linear with respect to the local coordinates $u^{i}$, and the Poisson bracket is linear with respect to the fields $u(x)$ :

$$
g^{i j \alpha}(u)=\left(b_{k}^{i j \alpha}+b_{k}^{j i \alpha}\right) u^{k}+g_{0}^{i j \alpha}, \quad b_{k}^{i j \alpha}=\mathrm{const}, \quad g_{0}^{i j \alpha}=\mathrm{const}, \quad 2 \leq \alpha \leq n .
$$

Let the metric $g^{i j 1}$ be constant. Then in these local coordinates we have $\Gamma_{j k}^{i 1}=0$. It follows from the relation (3.4) that in these coordinates $T^{i j k 1 \alpha}=$ const for all $\alpha$. Let us prove that all the coefficients $b_{k}^{i j \alpha}$ are also constant in these coordinates. Indeed, we have

$b_{k}^{i j \alpha}=-g^{i s \alpha} \Gamma_{s k}^{j \alpha}=-g^{i s \alpha} \Gamma_{k s}^{j \alpha}=-g^{i s \alpha} T_{k s}^{j 1 \alpha}=-g_{k q 1} g^{i s \alpha} g^{q r 1} T_{r s}^{j 1 \alpha}=-g_{k q 1} T^{q j i 1 \alpha}=$ const.

Here, we have used the fact that all the connections $\Gamma_{j k}^{i \alpha}$ are symmetric. Then the linearity of all the metrics in these local coordinates and the linearity of the bracket with respect to the fields follows from the relation (2.4).

For $N \geq 3$ Theorem 4.2 was proved by B.A.Dubrovin and S.P.Novikov in [1]. The study of the cases $N=1$ and $N=2$ in [1] is erroneous, since the study is based on an incomplete set of relations for the obstruction tensors, which is obtained in [1] and which is insufficient in order to guarantee that the bracket (2.1) is a Poisson bracket. In the complete form Theorem 4.2 was proved by the present author in 6].

Let us recall here very briefly, in a necessary for us form, a general scheme (see also [13]) that goes back to Sophus Lie and concerns interconnections between Lie 
algebras and Poisson structures whose coefficients depend linearly (possibly, nonhomogeneously) on coordinates (the Lie-Poisson brackets).

For the general infinite-dimensional case we shall describe special infinite-dimensional Lie algebras corresponding to arbitrary Poisson structures whose coefficients depend linearly (possibly, nonhomogeneously) on the field variables $u^{i}(x)$ and their derivatives $u_{(k)}^{i},(k)=\left(k_{1}, \ldots, k_{n}\right)$, where $u_{(k)}^{i}=\partial^{|k|} u^{i} /\left(\partial\left(x^{1}\right)^{k_{1}} \cdots \partial\left(x^{n}\right)^{k_{n}}\right),|k|=$ $k_{1}+\cdots+k_{n}$.

An operator $M^{i j}$ determining a Poisson bracket (a Poisson structure)

$$
\{I, J\}=\int \frac{\delta I}{\delta u^{i}(x)} M^{i j} \frac{\delta J}{\delta u^{j}(x)} d^{n} x
$$

is called Hamiltonian.

Consider arbitrary Hamiltonian operators given by differential operators whose coefficients depend linearly (generally speaking, nonhomogeneously) on the field variables $u^{i}(x)$ and their derivatives, i.e., Hamiltonian operators of the form

$$
M^{i j}=\left(a_{s}^{i j,(k)(p)} u_{(k)}^{s}+b^{i j,(p)}\right) \frac{d^{|p|}}{d\left(x^{1}\right)^{p_{1}} \cdots d\left(x^{n}\right)^{p_{n}}},
$$

where $a_{s}^{i j,(k)(p)}$ and $b^{i j,(p)}$ are constants.

Consider the infinite-dimensional space $S$ of sequences $\left(\xi_{1}, \ldots, \xi_{N}\right)$, where $\xi_{i} \in$ $C^{\infty}\left(T^{n}\right)$ are smooth functions on an $n$-dimensional torus. Then if $\xi$ and $\eta$ belong to the space $S$, we have

$$
(\xi, M(\eta)) \equiv \int_{T^{n}} \xi_{i} M^{i j} \eta_{j} d^{n} x=\int_{T^{n}} u^{s}[\xi, \eta]_{s} d^{n} x+\int_{T^{n}} \xi_{i} b^{i j,(p)} \eta_{j(p)} d^{n} x
$$

Thus on the space $S$ a bilinear operation $[\cdot, \cdot]$,

$$
(\xi, \eta) \mapsto \zeta=[\xi, \eta] \in S, \quad \zeta_{s}=[\xi, \eta]_{s}=\sum_{i, j,(k),(p)}(-1)^{|k|} a_{s}^{i j,(k)(p)}\left(\xi_{i} \eta_{j(p)}\right)_{(k)},
$$

and a bilinear form

$$
\omega(\xi, \eta)=\int_{T^{n}} \xi_{i} b^{i j,(p)} \eta_{j(p)} d^{n} x
$$

are defined.

An arbitrary operator $M^{i j}$ of the form (4.2) is skew-symmetric if and only if the corresponding bilinear operation (4.4) and bilinear form (4.5) are skew-symmetric on the space $S$, i.e., $[\xi, \eta]=-[\eta, \xi]$ and $\omega(\xi, \eta)=-\omega(\eta, \xi)$. 
An arbitrary operator $M^{i j}$ of the form (4.2) is Hamiltonian if and only if the corresponding space $S$ is a Lie algebra with respect to the corresponding bilinear operation (4.4), i.e., this bilinear operation is skew-symmetric and satisfies the Jacobi identity $[\xi,[\eta, \zeta]]+[\eta,[\zeta, \xi]]+[\zeta,[\xi, \eta]]=0$, and, in addition, the corresponding bilinear form (4.5) is a 2-cocycle on this Lie algebra, i.e., this bilinear form is skew-symmetric and satisfies the closedness identity

$$
(d \omega)(\xi, \eta, \zeta) \equiv \omega([\xi, \eta], \zeta)+\omega([\eta, \zeta], \xi)+\omega([\zeta, \xi], \eta)=0
$$

Note that a 2-cocycle $\omega(\xi, \eta)$ defined by a Hamiltonian operator of the form (4.2) is cohomologous to zero, i.e., $\omega(\xi, \eta)=(d f)(\xi, \eta) \equiv f([\xi, \eta])$, where $f$ is a 1 -form on the Lie algebra $S$, if and only if this 2-cocycle can be annihilated by a shift of the field variables $u^{i} \mapsto u^{i}-c^{i}$, where $c^{i}$ are arbitrary constants, i.e., provided that $b^{i j,(p)}=a_{k}^{i j,(0)(p)} c^{k}$.

Lemma 4.1 A multidimensional Poisson bracket of the form (2.1) is linear (possibly, nonhomogeneously) with respect to the fields $u(x)$ if and only if

$$
g^{i j \alpha}(u)=\left(b_{k}^{i j \alpha}+b_{k}^{j i \alpha}\right) u^{k}+g_{0}^{i j \alpha}, \quad b_{k}^{i j \alpha}=\mathrm{const}, \quad g_{0}^{i j \alpha}=\mathrm{const}, \quad 1 \leq \alpha \leq n,
$$

here the constants $b_{k}^{i j \alpha}$ and $g_{0}^{i j \alpha}$ satisfy nontrivial quadratic relations following from the relations (2.3) -(2.9) of Theorem 2.1 and define $n$ compatible algebras $\mathcal{B}^{\alpha}, 1 \leq \alpha \leq n$, of Frobenius and quasi-Frobenius type with ctructural constants $b_{k}^{i j \alpha}$ and symmetric bilinear forms $g_{0}^{i j \alpha}$ on these algebras (one can consider that there is one $N$-dimensional algebra with the basis $e^{1}, \ldots, e^{N}$ furnished with $n$ compatible multiplications and $n$ symmetric bilinear forms, $\left.e^{i} \stackrel{\alpha}{\circ} e^{j}=b_{k}^{i j \alpha} e^{k},\left\langle e^{i}, e^{j}\right\rangle_{\alpha}=g_{0}^{i j \alpha}, 1 \leq \alpha \leq n\right)$. To each multidimensional Poisson bracket of the form (2.1) that is linear (possibly, nonhomogeneously) with respect to the fields $u(x)$ it corresponds an infinite-dimensional Lie algebra of a special type with a 2-cocycle of a special type on it (a Lie algebra of hydrodynamic type):

$$
\begin{gathered}
{[\xi, \eta]_{k}=b_{k}^{i j \alpha}\left(\left(\eta_{i}\right)_{\alpha} \xi_{j}-\eta_{j}\left(\xi_{i}\right)_{\alpha}\right),} \\
b_{k}^{i j \alpha}=\text { const }, \quad \xi=\left(\xi_{1}, \ldots, \xi_{N}\right), \quad \xi_{i}(x) \in C^{1}\left(T^{n}\right), \\
\omega(\xi, \eta)=\int g_{0}^{i j \alpha}\left(\eta_{j}(x)\right)_{\alpha} \xi_{i}(x) d^{n} x, \quad g_{0}^{i j \alpha}=\text { const. }
\end{gathered}
$$

The classification of multidimensional Poisson brackets of the form (2.1) that are linear (possibly, nonhomogeneously) with respect to the fields $u(x)$ and the classification of related to them infinite-dimensional Lie algebras, admissible 2-cocycles 
on these Lie algebras, and also algebras $\mathcal{B}^{\alpha}, 1 \leq \alpha \leq n$, of Frobenius and quasiFrobenius type generating the brackets is a separate important problem, which is far from a complete solution for now.

In the one-dimensional case, this problem was solved by A.A.Balinsky and S.P.Novikov in [7], where, as is important to note, was discovered its connection with the theory of Frobenius and quasi-Frobenius algebras, although in the one-dimensional case also many questions remained unsolved.

In the multidimensional case for $n \geq 2$ this problem was studied by the present author in [6], where the theory of the corresponding algebras was developed and complete classification results were obtained for the cases, when the number of components is not more than 4 . The results on this problem and, in particular, on the theory of the corresponding quasi-Frobenius algebras will be published in a separate paper.

Theorem 4.3 Each nondegenerate multidimensional Poisson bracket of the form (2.1) is defined by a certain infinite-dimensional Lie algebra of the form (4.8), (4.9) with a certain 2-cocycle of the form (4.10) on this Lie algebra for which

$$
b_{k}^{i j 1}=0, \quad \operatorname{det}\left(\left(b_{k}^{i j \alpha}+b_{k}^{j i \alpha}\right) u^{k}+g_{0}^{i j \alpha}\right) \neq 0, \quad 1 \leq \alpha \leq n .
$$

Theorem 4.3 immediately follows from Theorem 4.2 and Lemma 4.1. The theory of infinite-dimensional Lie algebras of hydrodynamic type (4.8), (4.9), (4.10) and algebras $\mathcal{B}^{\alpha}$ with (and also without) the additional conditions (4.11) was developed by the present author in [6], where complete classification results were obtained for the cases, when the number of components is not more than 4 .

Example 4.1 A Poisson bracket generated by the Lie algebra of vector fields on an $n$-dimensional torus $T^{n}$.

In this case $N=n$. The commutator of vector fields $\xi$ and $\eta$ has the form

$$
[\xi, \eta]_{k}=\xi_{s} \frac{\partial \eta_{k}}{\partial x^{s}}-\eta_{s} \frac{\partial \xi_{k}}{\partial x^{s}}
$$

where $\xi=\left(\xi_{1}(x), \ldots, \xi_{n}(x)\right), \eta=\left(\eta_{1}(x), \ldots, \eta_{n}(x)\right), x=\left(x^{1}, \ldots, x^{n}\right), x \in T^{n}$.

Then for the corresponding Hamiltonian operator $M^{i j}$ we obtain

$$
\begin{aligned}
& \int_{T^{n}} u^{k}[\xi, \eta]_{k} d^{n} x=\int_{T^{n}} u^{k}\left(\xi_{i} \frac{\partial \eta_{k}}{\partial x^{i}}-\eta_{j} \frac{\partial \xi_{k}}{\partial x^{j}}\right) d^{n} x= \\
& =\int_{T^{n}} \xi_{i}\left(u^{i} \frac{d}{d x^{j}}+u^{j} \frac{d}{d x^{i}}+\frac{\partial u^{i}}{\partial x^{j}}\right) \eta_{j} d^{n} x=\int_{T^{n}} \xi_{i} M^{i j} \eta_{j} d^{n} x
\end{aligned}
$$




$$
\begin{gathered}
M^{i j}=u^{i} \frac{d}{d x^{j}}+u^{j} \frac{d}{d x^{i}}+\frac{\partial u^{i}}{\partial x^{j}}=\left(u^{i} \delta^{j \alpha}+u^{j} \delta^{i \alpha}\right) \frac{d}{d x^{\alpha}}+\delta_{k}^{i} \delta^{j \alpha} u_{\alpha}^{k}, \\
g^{i j \alpha}=u^{i} \delta^{j \alpha}+u^{j} \delta^{i \alpha}, \quad b_{k}^{i j \alpha}=\delta_{k}^{i} \delta^{j \alpha} .
\end{gathered}
$$

It follows from the relations of Theorem 2.1 that if for a 2-cocycle on the Lie algebra of vector fields on $T^{n}$ the corresponding Poisson structure remains in the class of Poisson structures of hydrodynamic type (2.1) (see formulae (4.3) and (4.5)), then this 2-cocycle is cohomologous to zero.

For $n \leq 2$ the Poisson structure (4.14) is nondegenerate, but for $n>2$ all the metrics in (4.14) are degenerate.

For $n=1$ the only metric has the form $g(u)=2 u$, and the Hamiltonian operator has the form

$$
M=2 u \frac{d}{d x}+u_{x}
$$

For $n=2$ both the metrics are nondegenerate and indefinite:

$$
\left(g^{i j 1}\right)=\left(\begin{array}{cc}
2 u^{1} & u^{2} \\
u^{2} & 0
\end{array}\right), \quad\left(g^{i j 2}\right)=\left(\begin{array}{cc}
0 & u^{1} \\
u^{1} & 2 u^{2}
\end{array}\right)
$$

and the Hamiltonian operator has the form

$$
\left(M^{i j}\right)=\left(\begin{array}{cc}
2 u^{1} & u^{2} \\
u^{2} & 0
\end{array}\right) \frac{d}{d x^{1}}+\left(\begin{array}{cc}
0 & u^{1} \\
u^{1} & 2 u^{2}
\end{array}\right) \frac{d}{d x^{2}}+\left(\begin{array}{cc}
u_{x^{1}}^{1} & u_{x^{2}}^{1} \\
u_{x^{1}}^{2} & u_{x^{2}}^{2}
\end{array}\right) .
$$

The Poisson structure (4.18) cannot be reduced to a constant form by a local change of coordinate, since the obstruction tensor $T^{i j k 12}$ is not equal to zero identically:

$$
\begin{aligned}
& T^{i j k \alpha \beta}=g^{k s \beta} g^{i r \alpha}\left(\Gamma_{r s}^{j \beta}-\Gamma_{r s}^{j \alpha}\right)=-g^{i r \alpha} b_{r}^{k j \beta}+g^{k s \beta} b_{s}^{i j \alpha}= \\
& =-g^{i k \alpha} \delta^{j \beta}+g^{k i \beta} \delta^{j \alpha}=-\left(u^{i} \delta^{k \alpha}+u^{k} \delta^{i \alpha}\right) \delta^{j \beta}+\left(u^{i} \delta^{k \beta}+u^{k} \delta^{i \beta}\right) \delta^{j \alpha}= \\
& =u^{i}\left(\delta^{j \alpha} \delta^{k \beta}-\delta^{j \beta} \delta^{k \alpha}\right)+u^{k}\left(\delta^{j \alpha} \delta^{i \beta}-\delta^{j \beta} \delta^{i \alpha}\right) .
\end{aligned}
$$

In particular, $T^{11212}=u^{1}$.

This example is connected to the two-dimensional Euler hydrodynamics of ideal incompressible fluid (with further reduction to divergence-free vector fields).

Theorem 4.4 [6]. If for $N=n=2$, for a nondegenerate Poisson structure of the form (2.1), the obstruction tensor $T_{j k}^{i 12}(u)$ is not equal to zero identically, i.e., 
this Poisson structure cannot be reduced to a constant form by a local change of coordinates, then it can be reduced to a canonical form generated by the flat metrics

$$
\left(g^{i j 1}\right)=\left(\begin{array}{cc}
1 & 0 \\
0 & -1
\end{array}\right), \quad\left(g^{i j 2}\right)=\left(\begin{array}{cc}
2 u^{2} & u^{1}+u^{2} \\
u^{1}+u^{2} & 2 u^{1}
\end{array}\right) .
$$

Both the metrics (4.20) are indefinite and therefore, in particular, if one of the metrics of a two-dimensional two-component nondegenerate Poisson bracket of hydrodynamic type is positive or negative definite, then this Poisson bracket can be reduced to a constant form. The Poisson structure generated by the canonical flat metrics (4.20) is connected to the Lie algebra of vector fields on a two-dimensional torus $T^{2}$.

By virtue of Theorem 4.1, it is obvious that the canonical Poisson structure generated by the metrics (4.20) cannot be reduced to a constant form by local changes of coordinates. The obstruction tensor $T^{i j k 12}$ of this Poisson structure is not equal to zero: $T^{i j k 12}=g^{k s 2} g^{i r 1}\left(\Gamma_{r s}^{j 2}-\Gamma_{r s}^{j 1}\right)=g^{k s 2} \varepsilon^{i} \delta^{i r} \Gamma_{r s}^{j 2}=g^{k s 2} \varepsilon^{i} \Gamma_{i s}^{j 2}=-\varepsilon^{i} b_{i}^{k j 2}, \varepsilon^{1}=1$, $\varepsilon^{2}=-1$, in particular, $T^{21112}=b_{2}^{112}=1$.

The Poisson structure

$$
\left\{w^{i}(x), w^{j}(y)\right\}=\left(w^{i}(x) \delta^{j \alpha}+w^{j}(x) \delta^{i \alpha}\right) \delta_{\alpha}(x-y)+\delta_{k}^{i} \delta^{j \alpha} w_{\alpha}^{k}(x) \delta(x-y)
$$

generated by the Lie algebra of vector fields on a two-dimensional torus $T^{2}$ (example 4.1) is reduced to the canonical form given by the metrics (4.20) by the following local quadratic change of coordinates [6]:

$$
w^{1}=\frac{1}{2}\left(\left(u^{1}\right)^{2}-\left(u^{2}\right)^{2}\right), \quad w^{2}=\frac{1}{2}\left(u^{1}+u^{2}\right) .
$$

Each two-dimensional two-component nondegenerate Poisson structure of hydrodynamic type either can be reduced to a constant form or is generated by the Lie algebra of vector fields on a two-dimensional torus $T^{2}$ [6].

For $n>2$ each multidimensional two-component nondegenerate Poisson structure of hydrodynamic type either can be reduced to a constant form or can be reduced to the two-dimensional canonical Poisson bracket given by the metrics (4.20) by a local change of coordinates and by an unimodular change of the independent space variables $x^{i}$ [6].

Note that each constant multidimensional (for an arbitrary dimension $n$ ) twocomponent Dubrovin-Novikov bracket is reduced by an unimodular change of the independent space variables $x^{i}$ to a constant Dubrovin-Novikov bracket whose dimension is not more than 3 (but it cannot be reduced, generally speaking, to a two-dimensional bracket). 


\section{Compatible metrics}

Two pseudo-Riemannian contravariant metrics $g_{1}^{i j}(u)$ and $g_{2}^{i j}(u)$ are called compatible if for any linear combination $g^{i j}(u)=\lambda_{1} g_{1}^{i j}(u)+\lambda_{2} g_{2}^{i j}(u)$ of these metrics, where $\lambda_{1}$ and $\lambda_{2}$ are arbitrary constants, the coefficients of the corresponding Levi-Civita connections and the components of the corresponding Riemannian curvature tensors are related by the same linear relation: $\Gamma_{k}^{i j}(u)=\lambda_{1} \Gamma_{1, k}^{i j}(u)+\lambda_{2} \Gamma_{2, k}^{i j}(u), R_{k l}^{i j}(u)=$ $\lambda_{1} R_{1, k l}^{i j}(u)+\lambda_{2} R_{2, k l}^{i j}(u)$ [8].

Indices of the coefficients of connections and indices of the curvature tensors are raised and lowered by the metrics corresponding to them: $\Gamma_{k}^{i j}(u)=g^{i s}(u) \Gamma_{s k}^{j}(u)$, $R_{k l}^{i j}(u)=g^{i s}(u) R_{s k l}^{j}(u)$.

If for any linear combination of metrics the above-mentioned relation only for the corresponding Levi-Civita connections is fulfilled, then the metrics are called almost compatible.

The theory of compatible and almost compatible metrics is constructed by the present author in the paper [8]. This theory is closely connected to the theory of compatible Poisson brackets of hydrodynamic type (local and nonlocal), the theory of Frobenius manifolds and integrable systems. Recall that Poisson brackets are called compatible if each their linear combination is a Poisson bracket [14].

Theorem 5.1 [9]. All metrics $g^{i j \alpha}(u), 1 \leq \alpha \leq n$, defining a multidimensional Poisson bracket of the form (2.1) are mutually compatible. All one-dimensional DubrovinNovikov brackets forming a multidimensional Poisson bracket (see Lemma 2.1) are also mutually compatible.

This important theorem immediately follows from the tensor relations of compatibility of metrics [8], [15]. For two arbitrary pseudo-Riemannian metrics $g^{i j \alpha}(u)$ and $g^{i j \beta}(u)$ consider an analog of the obstruction tensors that were defined above for flat metrics of multidimensional Dubrovin-Novikov brackets: $T_{j k}^{i \alpha \beta}(u)=\Gamma_{j k}^{i \beta}(u)-\Gamma_{j k}^{i \alpha}(u)$, $T^{i j k \alpha \beta}(u)=g^{k s \beta}(u) g^{i r \alpha}(u) T_{r s}^{j \alpha \beta}(u)$, where $\Gamma_{j k}^{i \alpha}(u)$ is the Levi-Civita connection generated by the metric $g^{i j \alpha}(u)$. Then we have the following theorem.

Theorem 5.2 [8], [15]. Any two pseudo-Riemannian metrics $g^{i j \alpha}(u)$ and $g^{i j \beta}(u)$ are compatible if and only if the relations (3.1) and (3.3) of Theorem 3.1 are fulfilled. Moreover, the condition of almost compatibility is equivalent to the relation (3.1).

Note that namely the relations (3.1) and (3.3) for flat metrics of multidimensional Poisson brackets of hydrodynamic type were found by B.A.Dubrovin and S.P.Novikov in [1]. 
Thus the description and the classification of multidimensional Poisson brackets of hydrodynamic type correspond to the description and the classification of an important special subclass of compatible one-dimensional Poisson brackets of hydrodynamic type. This subclass is singled out by the additional relations (3.2) and (3.4).

The problem of the description of compatible one-dimensional Poisson brackets of hydrodynamic type that is equivalent to the description of flat pencils of compatible metrics or, in other words, local quasi-Frobenius manifolds, plays an important role in the theory of integrable systems of hydrodynamic type, the theory of Frobenius manifolds, the modern differential geometry and mathematical physics (see [16]).

The problem of the description of all nonsingular pairs of compatible flat metrics was solved by the present author in [17], [18] (see also [15]), where were obtained the nonlinear equations describing all nonsingular pairs of compatible flat metrics (these nonlinear equations are a nontrivial nonlinear reduction of the Lamé equations defining all the curvilinear orthogonal coordinate systems in a pseudo-Euclidean space) and was proved the integrability of these nonlinear equations by the inverse scattering method, moreover, was found an explicit integration and linearization procedure, which reduces the integration of these nonlinear equations to the solution of linear problems (thus a local classification of semisimple quasi-Frobenius manifolds was also obtained); see also [8], where the results were announced. Afterwards for these nonlinear equations also a Lax pair was found in [19].

A pair of metrics $g_{1}^{i j}(u)$ and $g_{2}^{i j}(u)$ is called nonsingular if all eigenvalues of this pair of metrics, i.e., the roots of the equation $\operatorname{det}\left(g_{1}^{i j}(u)-\lambda g_{2}^{i j}(u)\right)=0$, are distinct (the situation of a pair of metrics in general position).

Theorem 5.3 [8]. If a pair of metrics $g_{1}^{i j}(u)$ and $g_{2}^{i j}(u)$ is nonsingular, then these metrics are compatible if and only if for the affinor $v_{j}^{i}(u)=g_{1}^{i s}(u) g_{2, s j}(u)$ the Nijenhuis tensor

$$
N_{i j}^{k}(u)=v_{i}^{s}(u) \frac{\partial v_{j}^{k}}{\partial u^{s}}-v_{j}^{s}(u) \frac{\partial v_{i}^{k}}{\partial u^{s}}+v_{s}^{k}(u) \frac{\partial v_{i}^{s}}{\partial u^{j}}-v_{s}^{k}(u) \frac{\partial v_{j}^{s}}{\partial u^{i}}
$$

vanishes (here $g_{2, s j}(u)$ is the corresponding covariant metric, $g_{2}^{i s}(u) g_{2, s j}(u)=\delta_{j}^{i}$ ).

In this case, by virtue of the Nijenhuis theorem [20] there exist local coordinates (generally speaking, complex) in which the affinor $v_{j}^{i}(u)$ is diagonal in a domain: $v_{j}^{i}(u)=\lambda^{i}(u) \delta_{j}^{i}$. Then, in these special local coordinates, both the metrics $g_{1}^{i j}(u)$ and $g_{2}^{i j}(u)$ are also necessarily diagonal. Indeed, the eigenvalues $\lambda^{i}(u), 1 \leq i \leq N$, of the affinor $v_{j}^{i}(u)$ coincide with the eigenvalues of the pair of metrics $g_{1}^{i j}(u)$ and $g_{2}^{i j}(u)$ and, by our condition, they are distinct (the pair of metrics is nonsingular): $\lambda^{i}(u) \neq \lambda^{j}(u)$ 
for $i \neq j$. Then, in the special local coordinates, in which the affinor $v_{j}^{i}(u)$ is diagonal in a domain, we have $g_{1}^{i j}(u)=\lambda^{i}(u) g_{2}^{i j}(u)$. By virtue of symmetry of metrics we obtain $\left(\lambda^{i}(u)-\lambda^{j}(u)\right) g_{2}^{i j}(u)=0$, therefore if $i \neq j$, then $g_{1}^{i j}(u)=g_{2}^{i j}(u)=0$, i.e., the metrics are diagonal. Moreover, the following important theorem is valid:

Theorem 5.4 [8]. If a pair of metrics $g_{1}^{i j}(u)$ and $g_{2}^{i j}(u)$ is nonsingular, then these metrics are compatible if and only if there exist local coordinates $u=\left(u^{1}, \ldots, u^{N}\right)$ (possibly, complex) such that $g_{2}^{i j}(u)=g^{i}(u) \delta^{i j}$ and $g_{1}^{i j}(u)=f^{i}\left(u^{i}\right) g^{i}(u) \delta^{i j}$, where $f^{i}\left(u^{i}\right), i=1, \ldots, N$, are functions of one variable (possibly, complex).

Note here that for an arbitrary pair of metrics the condition of vanishing the corresponding Nijenhuis tensor is equivalent to the condition of almost compatibility of the metrics, but not compatibility (there are corresponding counterexamples) [8].

\section{Classification theorem}

Theorem 6.1 If for a nondegenerate multidimensional Poisson bracket of the form (2.1) one of the metrics $g^{i j \alpha}(u)$ forms nonsingular pairs with all the remaining metrics of the bracket, then this Poisson bracket can be reduced to a constant form by a local change of coordinates.

Let us prove that all the obstruction tensors $T_{j k}^{i \alpha \beta}(u)$ are equal to zero identically. Without loss of generality we can consider that the metric $g^{i j 1}(u)$ forms nonsingular pairs with all the remaining metrics of the bracket. Let $\beta \neq 1$. By theorem 5.1 the metrics $g^{i j \beta}(u)$ and $g^{i j 1}(u)$ are compatible. By Theorem 5.4 there exist local coordinates $u=\left(u^{1}, \ldots, u^{N}\right)$ (possibly, complex) such that $g^{i j \beta}(u)=g^{i}(u) \delta^{i j}$ and $g^{i j 1}(u)=f^{i}\left(u^{i}\right) g^{i}(u) \delta^{i j}$, where $f^{i}\left(u^{i}\right), i=1, \ldots, N$, are functions of one variable (possibly, complex). In these local coordinates $\Gamma_{j k}^{i \beta}(u)=0$ if all the indices $i, j, k$ are distinct; $\Gamma_{i k}^{i \beta}(u)=\Gamma_{k i}^{i \beta}(u)=-\left(1 /\left(2 g^{i}(u)\right)\right)\left(\partial g^{i} / \partial u^{k}\right)$ for any indices $i, k ; \Gamma_{j j}^{i \beta}(u)=$ $\left(g^{i}(u) /\left(2\left(g^{j}(u)\right)^{2}\right)\right)\left(\partial g^{j} / \partial u^{i}\right)$ for $i \neq j$. For the obstruction tensor $T^{i j k 1 \beta}(u)$ in the local coordinates under consideration we obtain: $T^{i j k 1 \beta}(u)=0$ if all the indices $i, j, k$ are distinct; $T^{i j j 1 \beta}(u)=T^{i i j 1 \beta}(u)=0$ for $i \neq j ; T^{i i i 1 \beta}(u)=\left(\left(g^{i}(u)\right)^{2} / 2\right)\left(d f^{i}\left(u^{i}\right) / d u^{i}\right)$; $T^{i j i 1 \beta}(u)=\left(\left(f^{i}\left(u^{i}\right)-f^{j}\left(u^{j}\right)\right) / 2\right)\left(\partial g^{i} / \partial u^{j}\right)$ for $i \neq j$. From the relation (3.2) of Theorem 3.1 we obtain that $T^{i i i 1 \beta}=0, f^{i}\left(u^{i}\right)=\mu^{i}=$ const, and $T^{i j i 1 \beta}(u)=0$ for $i \neq j, g^{i}(u)=g^{i}\left(u^{i}\right)$ are functions of one variable. Thus $T^{i j k 1 \beta}(u)=0$ for any $\beta$. Since for all indices $\alpha$ and $\beta$ the identity $T_{j k}^{i \alpha \beta}(u)=T_{j k}^{i 1 \beta}(u)-T_{j k}^{i 1 \alpha}(u)$ holds, we obtain that $T_{j k}^{i \alpha \beta}(u)=0$ for all $\alpha$ and $\beta$. 
Thus all pairs of metrics defining Dubrovin-Novikov brackets that cannot be reduced to a constant form, in particular, all nonconstant canonical pairs of metrics (they are connected to nontrivial Lie algebras of hydrodynamic type and nontrivial quasi-Frobenius algebras), are nonsingular, i.e., they have coinciding eigenvalues. In particular, for the unique nonconstant canonical pair of two-component metrics (4.20) we have: $\operatorname{det}\left(g^{i j 2}-\lambda g^{i j 1}\right)=0, \lambda_{1}=\lambda_{2}=u^{2}-u^{1}$. The theory of singular nondegenerate multidimensional (and also all degenerate) Dubrovin-Novikov brackets and algebras related to them is far from completeness at present.

Acknowledgements. I gratefully acknowledge the hospitality of Max-PlanckInstitut für Mathematik in Bonn (Germany).

\section{References}

[1] Dubrovin B. A., Novikov S. P. On Poisson brackets of hydrodynamic type. Doklady Akademii Nauk SSSR, 279, No. 2, pp. 294-297 (1984); English translation in: Soviet Math. Dokl., 30, pp. 651-654 (1984).

[2] Dubrovin B. A., Novikov S. P. The Hamiltonian formalism of one-dimensional systems of hydrodynamic type and the Bogolyubov-Whitham averaging method. Doklady Akademii Nauk SSSR, 270, No. 4, pp. 781-785 (1983); English translation in: Soviet Math. Dokl., 27, pp. 665-669 (1983).

[3] Tsarev S. P. Geometry of Hamiltonian systems of hydrodynamic type. The generalized hodograph method. Izvestiya Akademii Nauk SSSR. Seriya Matematematicheskaya, 54, No. 5, pp. 1048-1068 (1990); English translation in: Math. USSR - Izvestiya, 54, No. 5, pp. 397-419 (1990).

[4] Dubrovin B. A., Novikov S. P. Hydrodynamics of weakly deformed soliton lattices. Differential geometry and Hamiltonian theory. Uspekhi Matematicheskikh Nauk, 44, No. 6, pp. 29-98 (1989); English translation in: Russian Mathematical Surveys, 44, No. 6, pp. 35-124 (1989).

[5] Ferapontov E. V., Khusnutdinova K. R. On the integrability of $(2+1)$ dimensional quasilinear systems. Commun. Math. Phys., 248, 187-206 (2004); http://arXiv.org/nlin.SI/0305044 (2004). 
[6] Mokhov O. I. On Poisson brackets of Dubrovin-Novikov type (DN-brackets). Funktsional'nyi Analiz i Ego Prilozheniya, 22, No. 4, pp. 92-93 (1988); English translation in: Functional Analysis and Its Applications, 22, No. 4, pp. 336-338 (1988).

[7] Balinskii A. A., Novikov S. P. Poisson brackets of hydrodynamic type, Frobenius algebras and Lie algebras. Doklady Akademii Nauk SSSR, 283, No. 5, pp. 1036 1039 (1985); English translation in: Soviet Math. Dokl., 32, pp. 228-231 (1985).

[8] Mokhov O. I. Compatible and almost compatible pseudo-Riemannian metrics. Funktsional'nyi Analiz i Ego Prilozheniya, 35, No. 2, pp. 24-36 (2001); English translation in: Functional Analysis and Its Applications, 35, No. 2, pp. 100-110 (2001); http://arXiv.org/math.DG/0005051 (2000).

[9] Mokhov O. I. Symplectic and Poisson structures on loop spaces of smooth manifolds and integrable systems. Uspekhi Matematicheskikh Nauk, 53, No. 3, pp. 85-192 (1998); English translation in: Russian Mathematical Surveys, 53, No. 3 , pp. 515-622 (1998).

[10] Dorfman I. Dirac structures and integrability of nonlinear evolution equations. John Wiley \& Sons. Chichester, 1993.

[11] Grinberg N. I. On Poisson brackets of hydrodynamic type with a degenerate metric. Uspekhi Matematicheskikh Nauk, 40, No. 4, pp. 217-218 (1985); English translation in: Russian Mathematical Surveys, 40, No. 4, pp. 231-232 (1985).

[12] Mokhov O. I. Hamiltonian systems of hydrodynamic type and constant curvature metrics. Phys. Lett. A, 166, Nos. 3, 4, pp. 215-216 (1992).

[13] Gelfand I. M., Dorfman I. Ya. Hamiltonian operators and infinite-dimensional Lie algebras. Funktsional'nyi Analiz i Ego Prilozheniya, 15, No. 3, pp. 23-40 (1981); English translation in: Functional Analysis and Its Applications, 15, No. 3, pp. 173-187 (1981).

[14] Magri F. A simple model of the integrable Hamiltonian equation. J. Math. Phys., 19, No. 5, pp. 1156-1162 (1978).

[15] Mokhov O. I. Compatible flat metrics. Journal of Applied Mathematics, 2, No. 7, pp. 337-370 (2002); http://arXiv.org/math.DG/0201224 (2002). 
[16] Dubrovin B. Geometry of 2D topological field theories. In: Integrable Systems and Quantum Groups, Lecture Notes in Math., Vol. 1620, Springer-Verlag, Berlin, pp. 120-348 (1996); http://arXiv.org/hep-th/9407018 (1994).

[17] Mokhov O. I. On integrability of the equations for nonsingular pairs of compatible flat metrics. Teoreticheskaya i Matematicheskaya Fizika, 130, No. 2, pp. 233-250 (2002); English translation in: Theoretical and Mathematical Physics, 130, No. 2, pp. 198-212 (2002); http://arXiv.org/math.DG/0005081 (2000).

[18] Mokhov O. I. Flat pencils of metrics and integrable reductions of the Lamé equations. Uspekhi Matematicheskikh Nauk, 56, No. 2, pp. 221-222 (2001); English translation in: Russian Mathematical Surveys, 56, No. 2, pp. 416-418 (2001).

[19] Ferapontov E. V. Compatible Poisson brackets of hydrodynamic type. J. Phys. A: Math. Gen., 34, pp. 2377-3388 (2001); http://arXiv.org/math.DG/0005221 (2000).

[20] Nijenhuis A. $X_{n-1}$-forming sets of eigenvectors. Indagationes Mathematicae, 13, No. 2, pp. 200-212 (1951). 